\title{
Opening of an alternative ion permeation pathway in a nociceptor TRP channel
}

Authors: Joris Vriens ${ }^{1,2, *}$, Katharina Held ${ }^{1,2,3}$, Annelies Janssens ${ }^{1,3}$, Balázs István Tóth ${ }^{1,3}$, Sara Kerselaers ${ }^{1}$, Bernd Nilius ${ }^{1}$, Rudi Vennekens ${ }^{1}$ and Thomas Voets ${ }^{1, *}$

\section{Affiliations:}

${ }^{1}$ Laboratory of Ion Channel Research and TRP Research Platform Leuven (TRPLe), KU Leuven, Herestraat 49 box 802, B-3000 Leuven, Belgium

${ }^{2}$ Laboratory of Obstetrics and Experimental Gynaecology, KU Leuven, Herestraat 49 box 611, B-3000 Leuven, Belgium

${ }^{3}$ These authors contributed equally.

*Correspondence to: Thomas.Voets@med.kuleuven.be or Joris.Vriens@med.kuleuven.be 


\section{Supplementary results:}

a
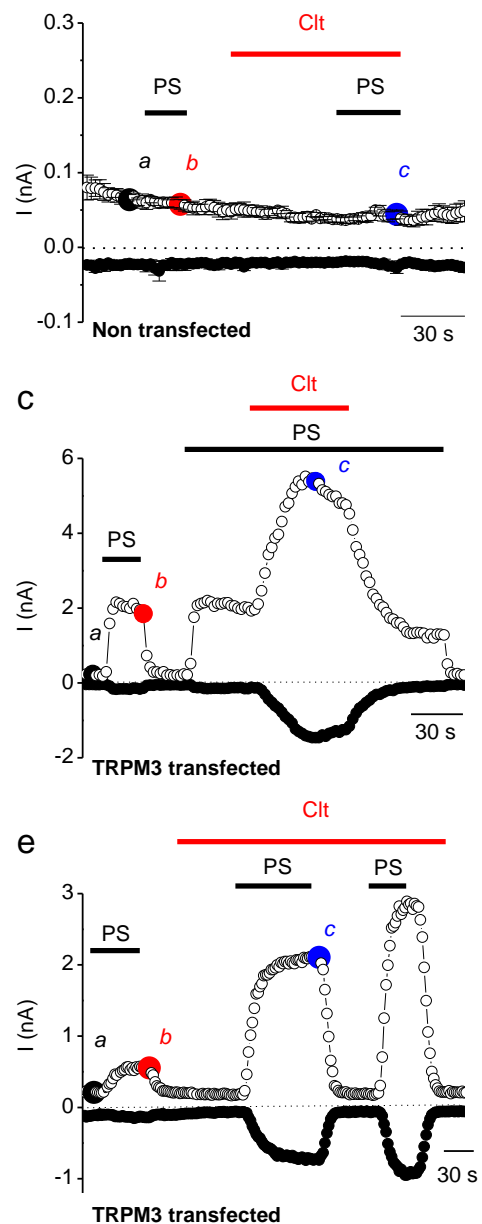

b

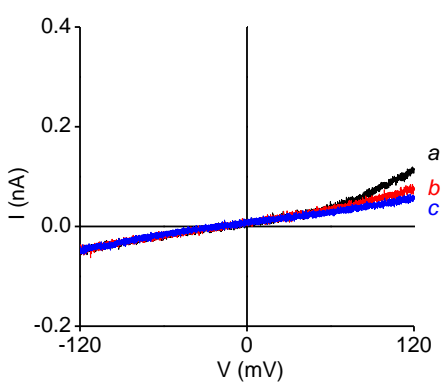

d

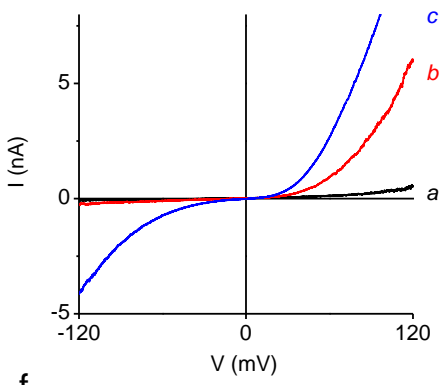

$f$

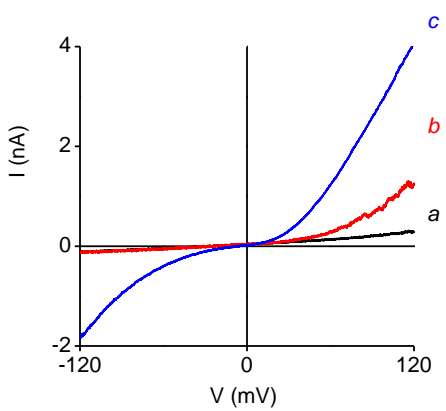

Supplementary Figure 1 Kinetics of the potentiating effect of Clt on PS-activated TRPM3 currents. (a) Mean time course of whole cell currents at $\pm 80 \mathrm{mV} \pm$ sem in non transfected HEK293 cells after stimulation by PS $(40 \mu \mathrm{M})$ and Clt $(10 \mu \mathrm{M})(\mathrm{n}=5)$. (b) Representative I-V relationship of currents at different time points as indicated in a. (c) Time course of whole cell currents at $\pm 80 \mathrm{mV}$ in HEK293 cells stably transfected with TRPM3 after stimulation by PS $(40 \mu \mathrm{M})$ and Clt $(10 \mu \mathrm{M})$. (d) I-V relationship of TRPM3 currents at different time points as indicated in c. (e) Time course of whole cell currents at $\pm 80 \mathrm{mV}$ after stimulation by PS, Clt and PS+Clt. (f) I-V relationship of TRPM3 currents at different time points as indicated in e. 

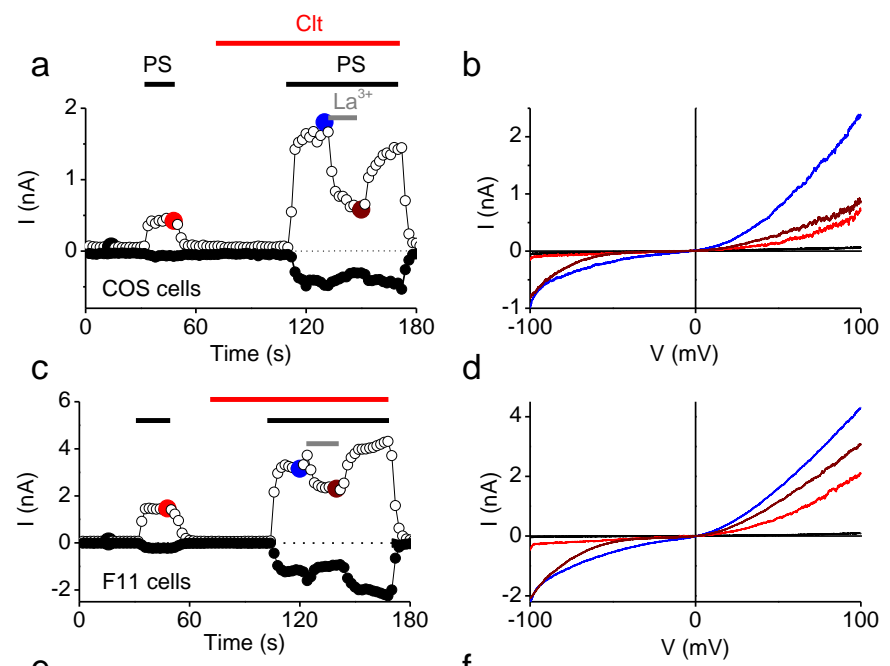

$f$
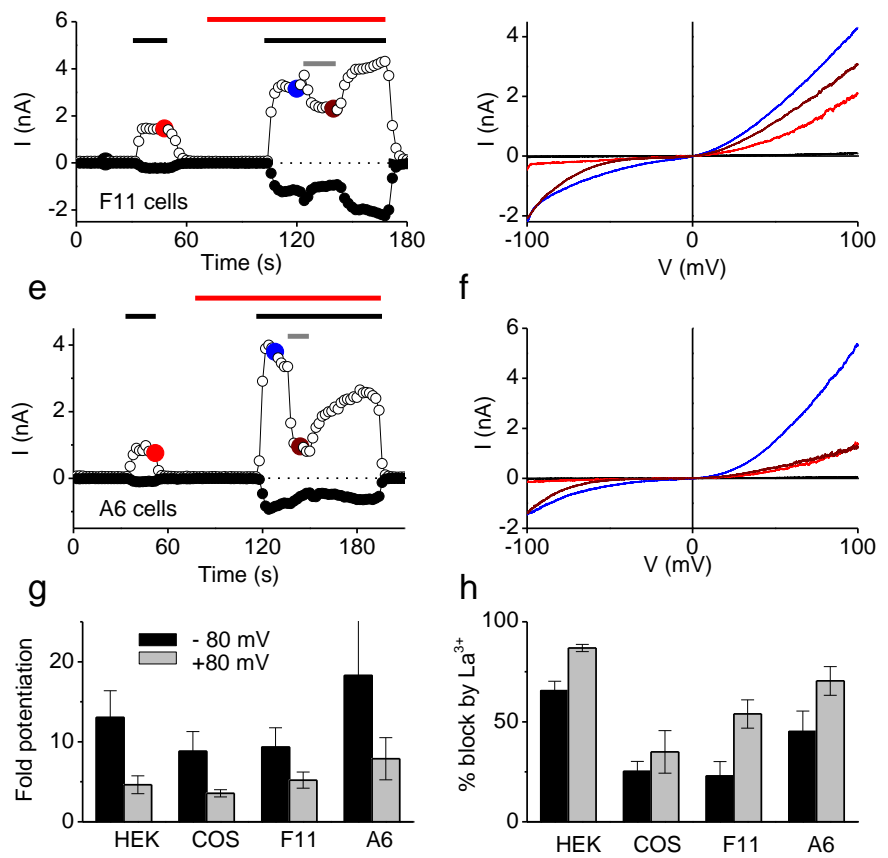

Supplementary Figure 2 Potentiation of PS by Clt in different expression systems. (a) Time course of whole cell currents at $\pm 80 \mathrm{mV}$ stimulated with PS $(40 \mu \mathrm{M})$, Clt $(10 \mu \mathrm{M})$ and $\mathrm{La}^{3+}$ $(10 \mu \mathrm{M})$ in COS cells. (b) I-V relations obtained at time points indicated in a. (c) Time course of whole cell currents at $\pm 80 \mathrm{mV}$ stimulated with PS $(40 \mu \mathrm{M})$, Clt $(10 \mu \mathrm{M})$ and $\mathrm{La}^{3+}$ $(10 \mu \mathrm{M})$ in the immortalized neuronal F11 cells transiently expressing TRPM3. (d) I-V relations obtained at time points indicated in c. (e,f) Identical protocol as described in $\mathbf{a}$ in the amphibian, A6 cells, a non-mammalian cell line transiently expressing TRPM3. (g) Ratio. 150/+150 for currents activated by PS and PS+Clt for HEK ( $n=84)$, neuronal F11 cells $(n=5)$ and amphibian A6 cells $(\mathrm{n}=4)$. (h) Percentage block by $\mathrm{La}^{3+}(10 \mu \mathrm{M})$ of the outward $(+100 \mathrm{mV})$ current activated after stimulation by PS+Clt in HEK ( $n=9)$, neuronal F11 (n=5) and amphibian A6 ( $\mathrm{n}=4)$ cells. 

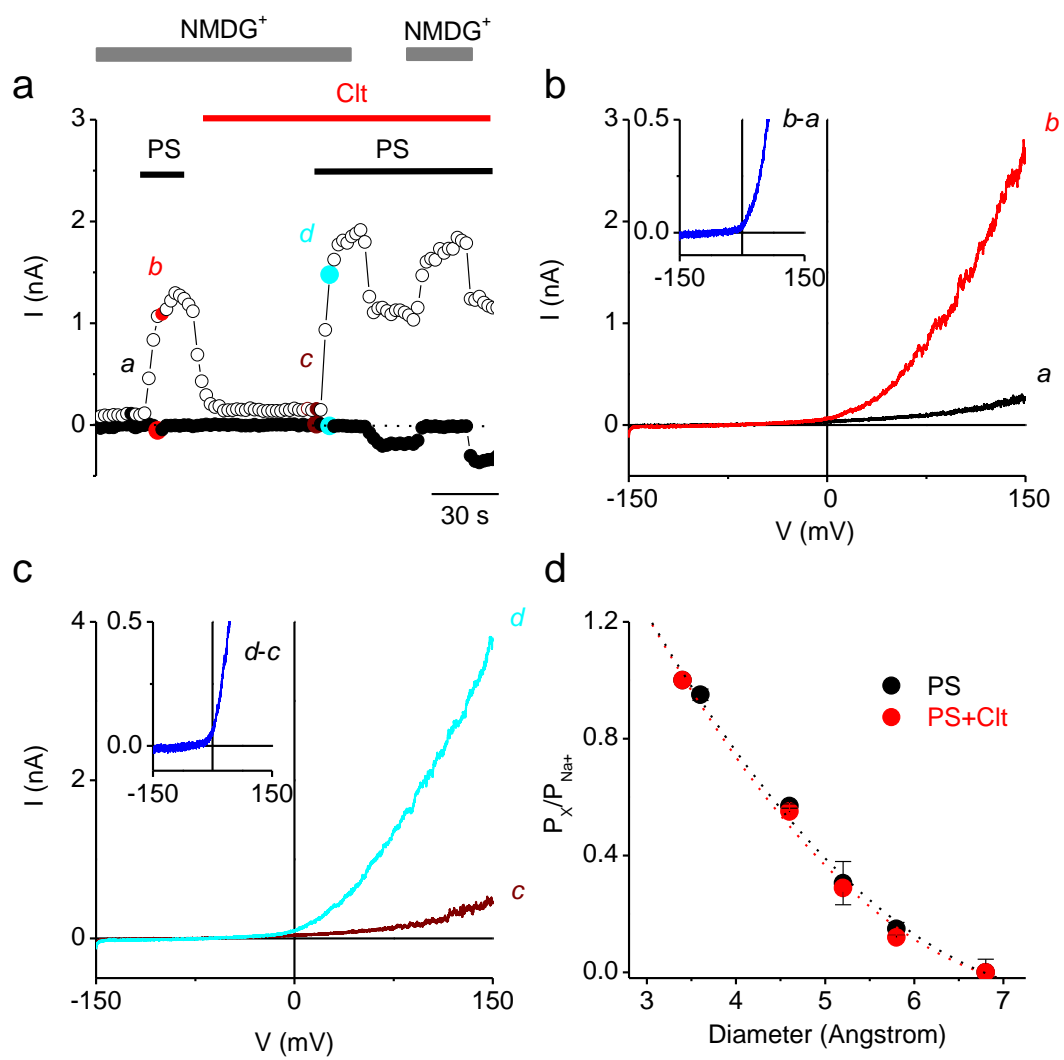

Supplementary Figure 3 Permeability properties of PS-activated TRPM3 currents in the absence and presence of Clt. (a) Time course of whole cell current at $\pm 80 \mathrm{mV}$ after stimulation by PS $(40 \mu \mathrm{M})$ and Clt $(10 \mu \mathrm{M})$. All $\mathrm{Na}^{+}$ions were replaced by $\mathrm{NMDG}^{+}$at indicated time points (gray boxes). (b) I-V traces at different time points as indicated in panel a. The inset shows the I-V relationship of the PS-activated current. (c) I-V traces at different time points as indicated in panel a. The inset shows the I-V relationship of the PS + Cltactivated current. (d) Shown are the relative permeabilities $\left(\boldsymbol{P}_{X} / \boldsymbol{P}_{\mathrm{Na}}\right)$ of different organic cations plotted as a function of the cation diameter for TRPM3 in presence of PS (black) and combined PS+Clt (red). Sodium was replaced by respectively monomethyl- (diameter: $3.6 \AA$ ), dimethyl- (diameter: $4.6 \AA$ A), trimethyl- (diameter: $5.2 \AA$ ), tetramethylammonium (diameter: $5.8 \AA$ ) and NMDG (diameter: $6.8 \AA$ ) $(\mathrm{n}=4)$. Dotted lines represent fits using the excludedvolume function (see Methods). 

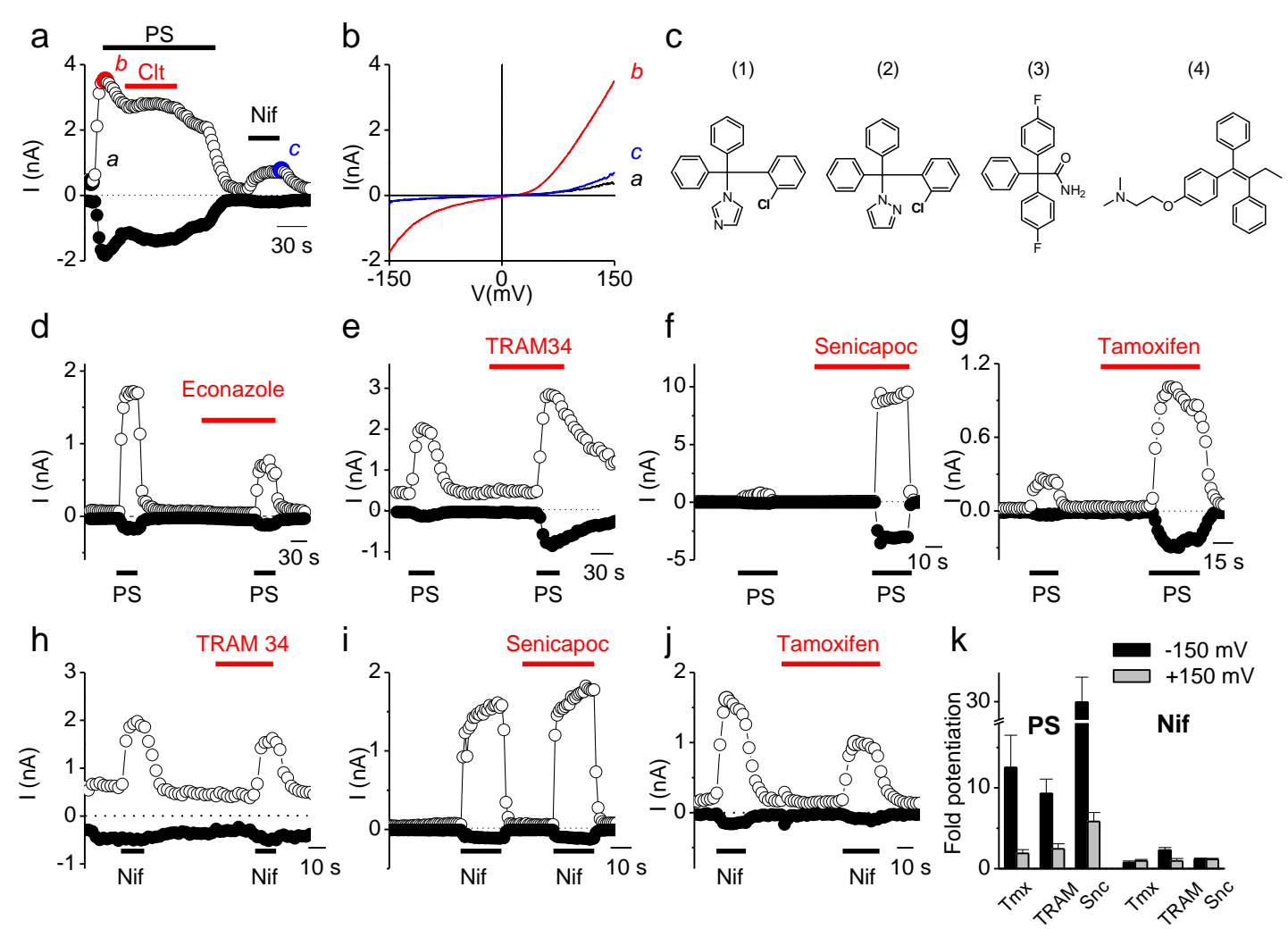

Supplementary Figure $4 \mathrm{Clt}$ and analogues potentiate PS-activated TRPM3 currents. (a) Time course of whole cell current at $\pm 150 \mathrm{mV}$ in stable transfected TRPM3 cells after stimulation by PS $(40 \mu \mathrm{M})$, PS+Clt $(10 \mu \mathrm{M})$ and nifedipine $(50 \mu \mathrm{M})$ with Clt $(10 \mu \mathrm{M})$ in the pipette solution. (b) I-V traces at different time points as indicated in panel a. (c) Chemical structures of (1) clotrimazole, (2) TRAM34, (3) Senicapoc and (4) Tamoxifen. (d) Time course of whole cell current at $\pm 80 \mathrm{mV}$ during stimulation with a PS $(40 \mu \mathrm{M})$, either alone or in combination with econazole $(10 \mu \mathrm{M})$. (e) Time course of whole cell current at $\pm 80 \mathrm{mV}$ during stimulation with a PS $(40 \mu \mathrm{M})$, either alone or in combination with TRAM-34 (10 $\mu \mathrm{M}$ ). (f) Time course of whole cell current at $\pm 80 \mathrm{mV}$ in transfected TRPM3 cells after stimulation by senicapoc $(20 \mu \mathrm{M})$ and the combined stimuli senicapoc+PS. (g) Time course of whole cell current at $\pm 80 \mathrm{mV}$ in transfected TRPM3 cells after stimulation by Tamoxifen (50 $\mu \mathrm{M})$ and the combined stimuli Tamoxifen+PS. (h-j) Time course of whole cell current at \pm 80 
$\mathrm{mV}$ in stable transfected TRPM3 cells after stimulation by nifedipine $(50 \mu \mathrm{M})$ and the combined stimuli TRAM34 (h), senicapoc (i), Tamoxifen (j) + nifedipine. (k) Potentiation by Tamoxifen (Tmx), TRAM34 (TRAM) and senicapoc (snc) of the inward (-150 mV, black bars) and outward (+150 mV, grey bars) current induced by PS (40 $\mu \mathrm{M})$ and nifedipine (50 $\mu \mathrm{M})$ in stable transfected TRPM3 cells (mean \pm sem; $n \geq 4$ ). 
a

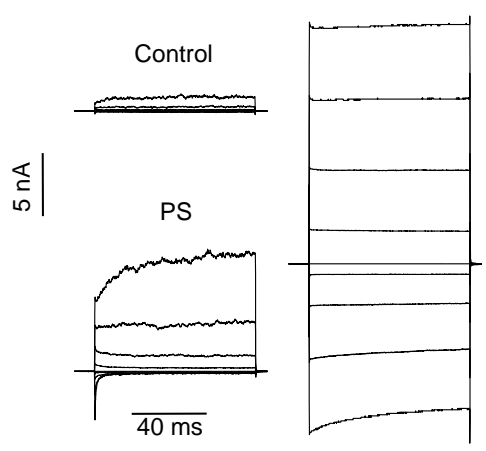

C

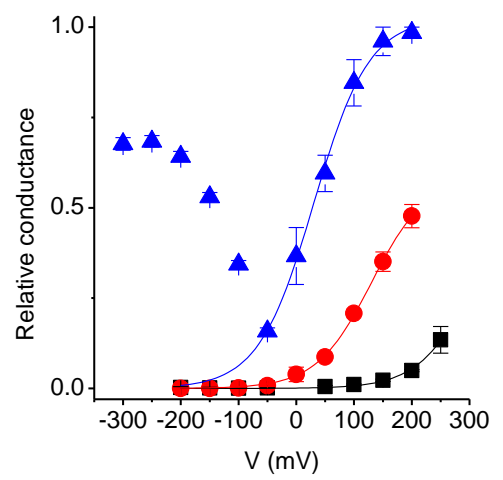

b

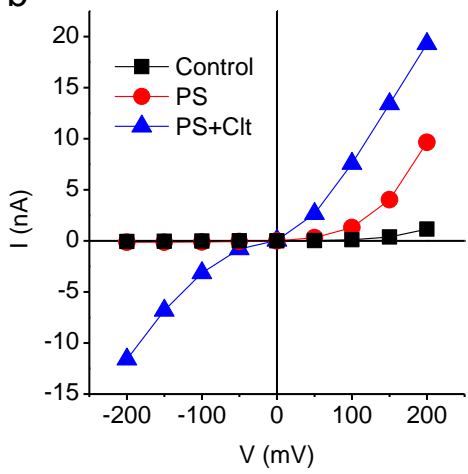

d

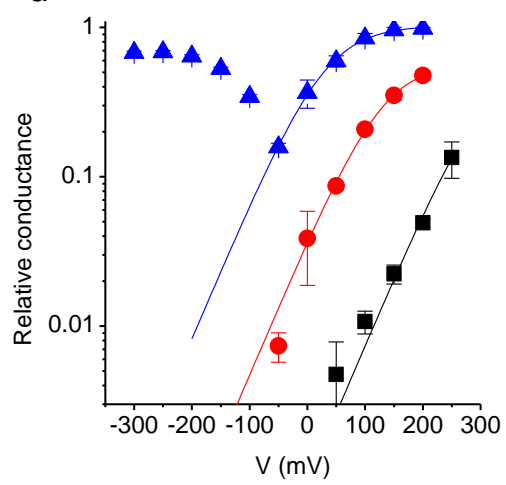

Supplementary Figure 5 Voltage dependence of TRPM3. (a) Whole-cell currents in response to a voltage step protocol (100-ms steps from $-200 \mathrm{mV}$ to +200 in $50-\mathrm{mV}$ increments) in TRPM3 transfected HEK293 cells in presence of standard bath solution (black), PS (40 $\mu \mathrm{M}$; red) and PS + Clt $(10 \mu \mathrm{M}$; green). (b) Steady-state I-V relationships obtained from currents in a. (c,d) Steady-state G-V relationships, with conductance normalized to the maximal conductance at $+200 \mathrm{mV}$ in the presence of PS + Clt $(n=6)$. Solid lines represent a global fit of a Boltzmann function (see methods) to the data points at voltages $\geq-50 \mathrm{mV}$, assuming constant gating charge $(\mathrm{z}=0.55)$. Values for $\mathrm{V}_{1 / 2}$ changed from $+251 \mathrm{mV}$ (control) to $+130 \mathrm{mV}$ (PS) and $+30 \mathrm{mV}$ (PS $+\mathrm{Clt})$. This analysis indicates that the effect of PS and PS+Clt on outward conductance can be largely explained as a leftward shift of the G-V curve, whereas the inward conductance in the combined presence of PS and Clt clearly deviates from this model. 

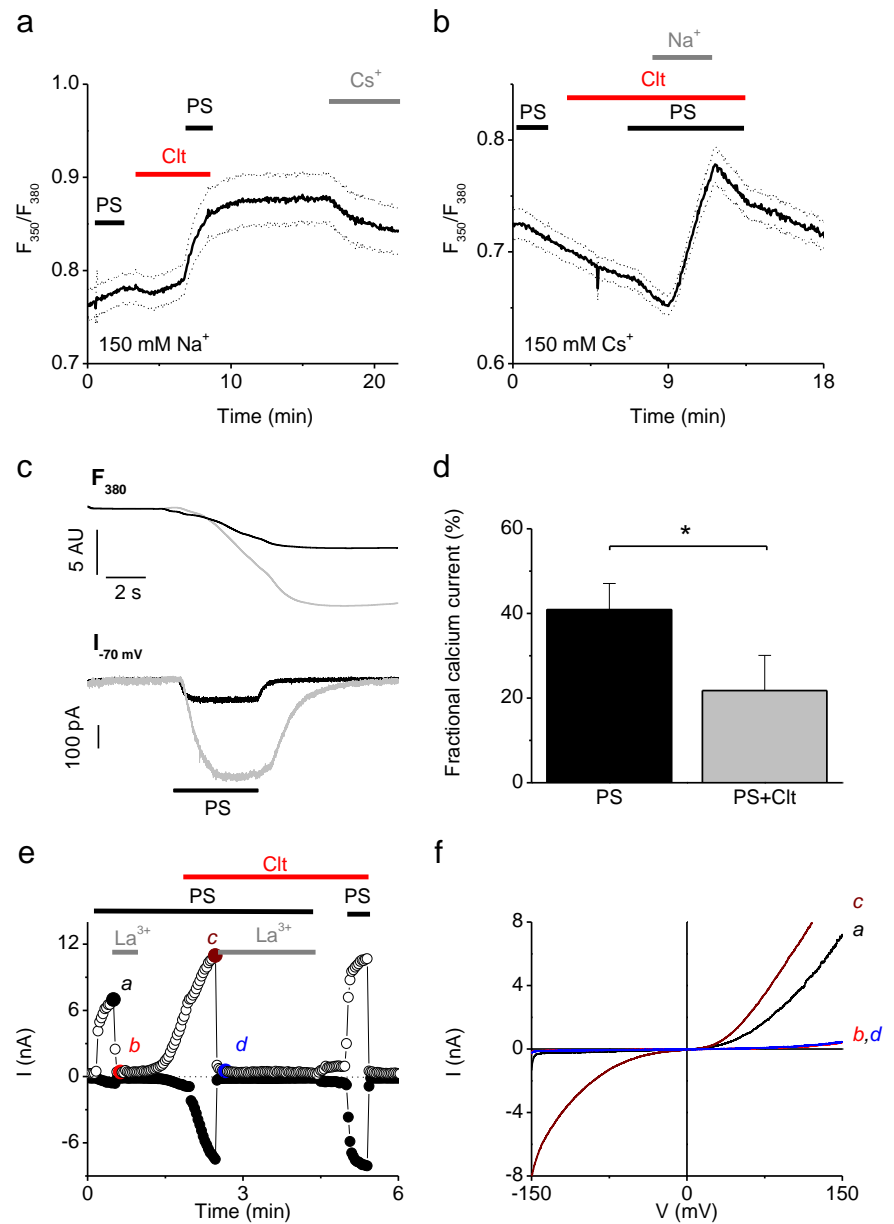

Supplementary Figure 6 Further properties of the alternative pathway. (a) Ratiometric imaging of TRPM3-expressing HEK293 cells loaded with SBFI. Cells are stimulated by PS $(40 \mu \mathrm{M})$ and Clt $(10 \mu \mathrm{M})(\mathrm{n}=33$ from at least three independent measurements). At indicated time (gray line) all extracellular $\mathrm{Na}^{+}$is replaced by $\mathrm{Cs}^{+}$. (b) Similar protocol as in a, but 150 $\mathrm{mM} \mathrm{CsCl}$ was used as extracellular salt. At the indicated time all extracellular $\mathrm{Cs}^{+}$is replaced by $\mathrm{Na}^{+}$. (c) Fractional $\mathrm{Ca}^{2+}$ current measurements. Upper panel illustrates Fura 380 measurements, exhibiting a decrease in signal that is linearly correlated to the amount of calcium influx. Lower panel shows simultaneous measurements of ionic currents at $-75 \mathrm{mV}$ in HEK293 cells stably expressing TRPM3. Cells were stimulated by PS $(40 \mu \mathrm{M})$ in the absence (black traces) or presence of Clt $(10 \mu \mathrm{M}$; gray traces). (d) Effect of Clt on the fractional calcium $\mathrm{Ca}^{2+}$ current of PS-induced TRPM3 currents $(\mathrm{p}=0.01$ for $\mathrm{n}=6$ different experiments). (e) Time course of whole cell currents at $\pm 150 \mathrm{mV}$ of TRPM3-expressing 
HEK293 cells after stimulation by PS $(40 \mu \mathrm{M})$, Clt $(10 \mu \mathrm{M})$ and the combined stimuli PS+Clt. All extracellular $\mathrm{NaCl}$ was replaced by $\mathrm{LaCl}_{3}(75 \mathrm{mM})$ at indicated time (gray box). (f) $\mathrm{I}-\mathrm{V}$ relations obtained at time points indicated in $\mathbf{e}$. 

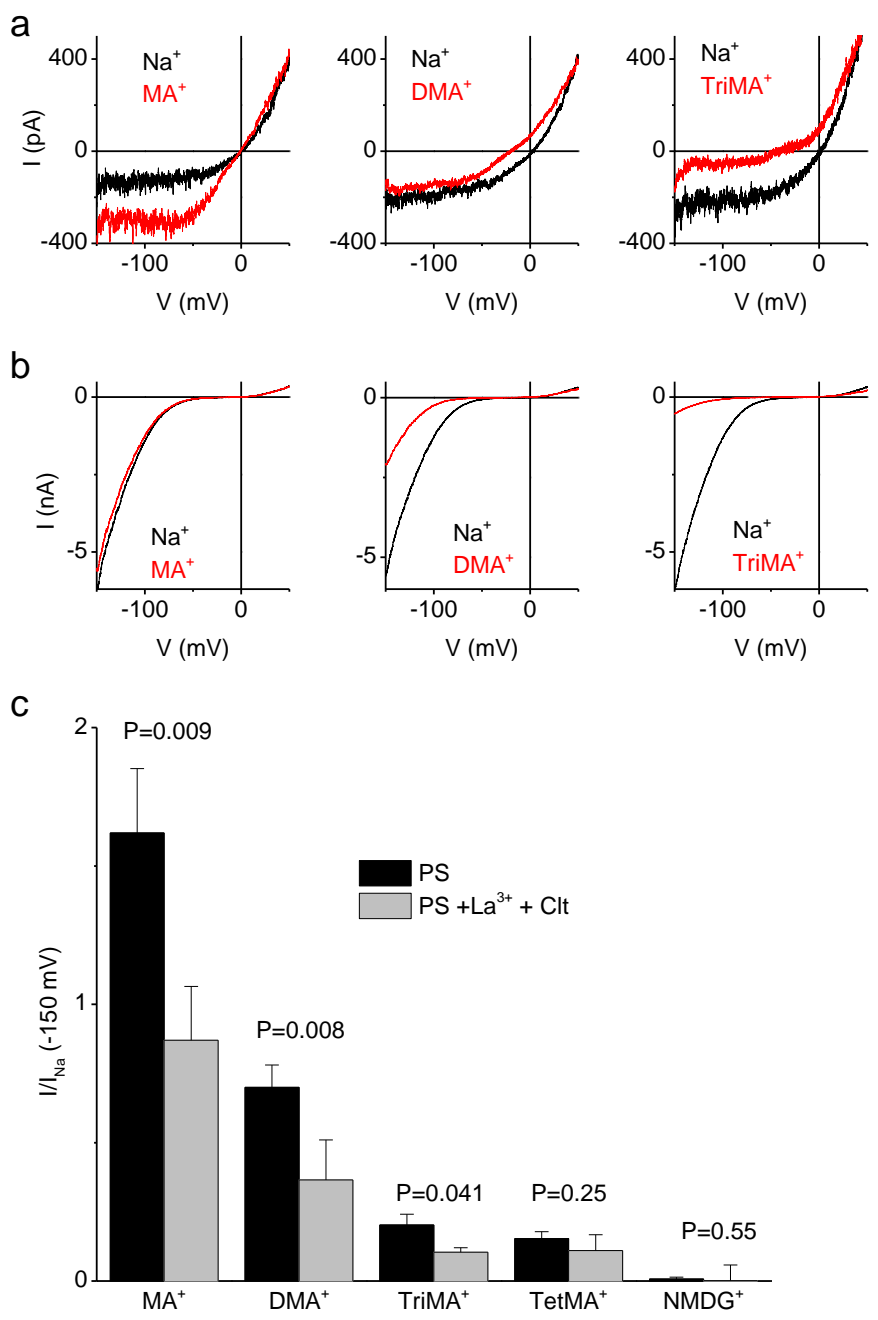

Supplementary Figure 7 Comparison of the permeability of the canonical and alternative pathway for organic cations. (a) TRPM3 currents activated by PS (40 $\mu \mathrm{M})$ before (black) and during (red) substitution of all external $\mathrm{Na}^{+}$ions by the indicated substituted ammonium ion. Currents recorded in the absence of PS were subtracted to isolated the TRPM3-dependent current. (b) As in a, but for TRPM3 currents recorded in the combined presence of PS (40 $\mu \mathrm{M})$, Clt $(10 \mu \mathrm{M})$ and $\mathrm{La}^{3+}(10 \mu \mathrm{M})$. (c) Comparison of the inward current at $-150 \mathrm{mV}$ (relative to $\mathrm{Na}^{+}$) carried by the indicated cations under the conditions of $\mathbf{a}$ and $\mathbf{b}$. Results from 7 cells for each condition. 

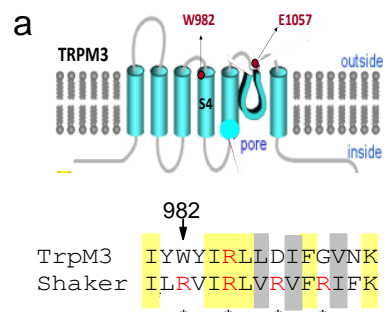

d
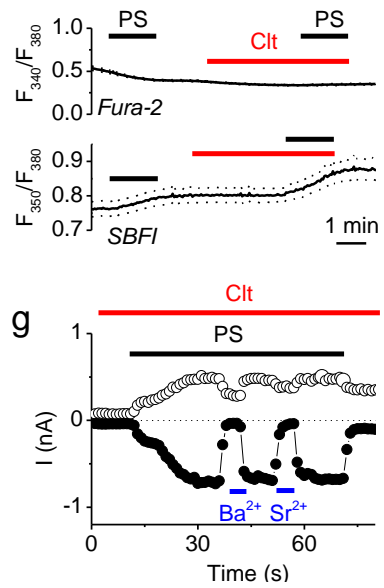
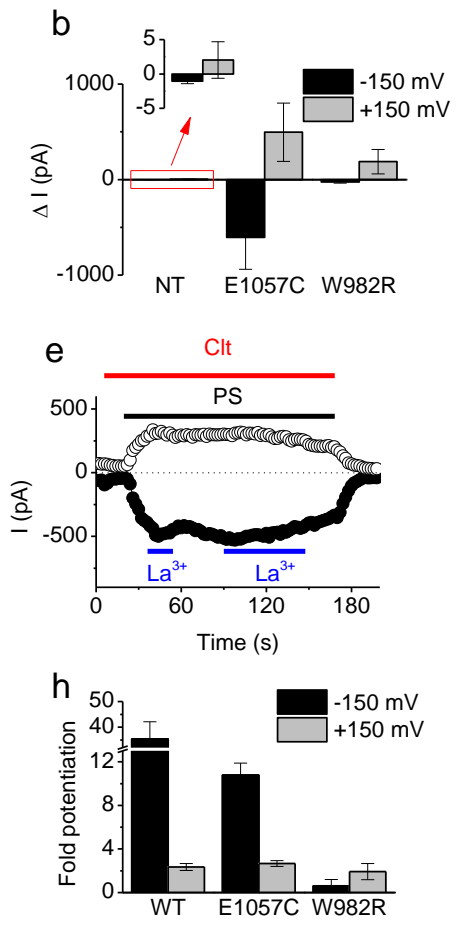

C
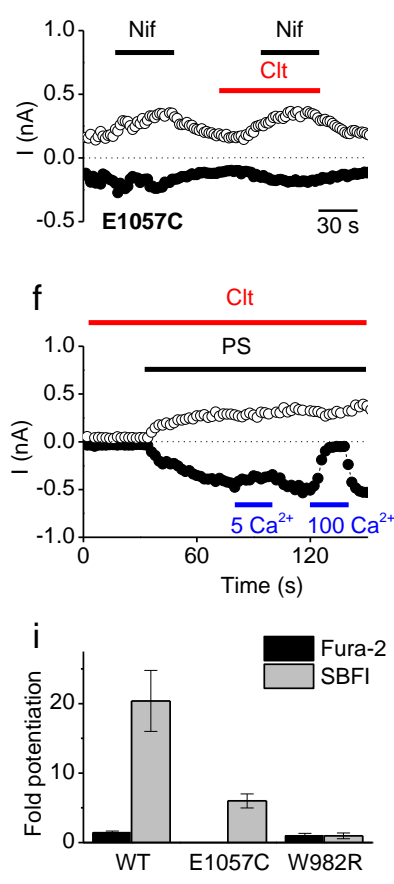

Supplementary Figure 8 Further properties of TRPM3 mutants. (a) Upper panel: cartoon illustrating the putative location of the different mutants. W982 is located in the putative transmembrane 4 domain (S4), E1057 is located in the putative pore region of TRPM3. Lower panel: alignment between the putative S4 segment of TRPM3 and the voltage sensor (S4) of the Shaker $\mathrm{K}^{+}$channel. Yellow background marks identical residues; grey background marks conserved residues. Arginines crucial for voltage sensing in Shaker are indicated with asterisks. (b) Current increase at $\pm 150 \mathrm{mV}$ upon stimulation with PS $(40 \mu \mathrm{M})+$ Clt $(10 \mu \mathrm{M})$ in non-transfected (NT) HEK293 cells ( $n=7)$, and HEK293 cells transfected with E1057C ( $n=$ 18) and W982R $(n=7)$. The inset shows the results for non-transfected cells at enlarged scale. (c) Time course of whole cell current at $\pm 80 \mathrm{mV}$ in E1057C transfected cells upon stimulation with nifedipine $(100 \mu \mathrm{M})$ and the combined stimuli Clt+nif. (d) Average changes in fluometric Fura-2 (upper panel) and SBFI (lower panel) measurements in response to PS (40 $\mu \mathrm{M})$, Clt $(10 \mu \mathrm{M})$ and Clt+PS in E1057C transfected HEK293T cells ( $>35$ from three independent measurements). No response was observed in non-transfected HEK293 cells 
(data not shown). (e) Time course of whole cell current at $\pm 80 \mathrm{mV}$ evoked by PS (40 $\mu \mathrm{M})$ and Clt $(10 \mu \mathrm{M})$ in a E1057C-expressing HEK293 cell, illustrating the lack of inhibition by $\mathrm{La}^{3+}$ $(100 \mu \mathrm{M})$. (f) As in e, illustrating the effect of adding $5 \mathrm{mM} \mathrm{Ca}^{2+}$ to the external solution or full substitution of all extracellular $\mathrm{NaCl}$ by $\mathrm{CaCl}_{2}(100 \mathrm{mM})$. (g) As in $\mathbf{f}$, but all extracellular $\mathrm{NaCl}$ was replaced by $\mathrm{BaCl}_{2}(100 \mathrm{mM})$ or $\mathrm{SrCl}_{2}(100 \mathrm{mM})$. (h) Relative potentiation by Clt of the inward $(-150 \mathrm{mV})$ and outward $(+150 \mathrm{mV})$ PS-activated current in $\mathrm{WT}(\mathrm{n}=84)$ and E1057C ( $\mathrm{n}=11)$ and W982R $(\mathrm{n}=7)$. (i) Relative potentiation by Clt of PS-induced increases in intracellular $\mathrm{Ca}^{2+}$ (Fura-2) and $\mathrm{Na}^{+}(\mathrm{SBFI}) \quad(\mathrm{n}>40$ cells from at least 3 independent measurements). 

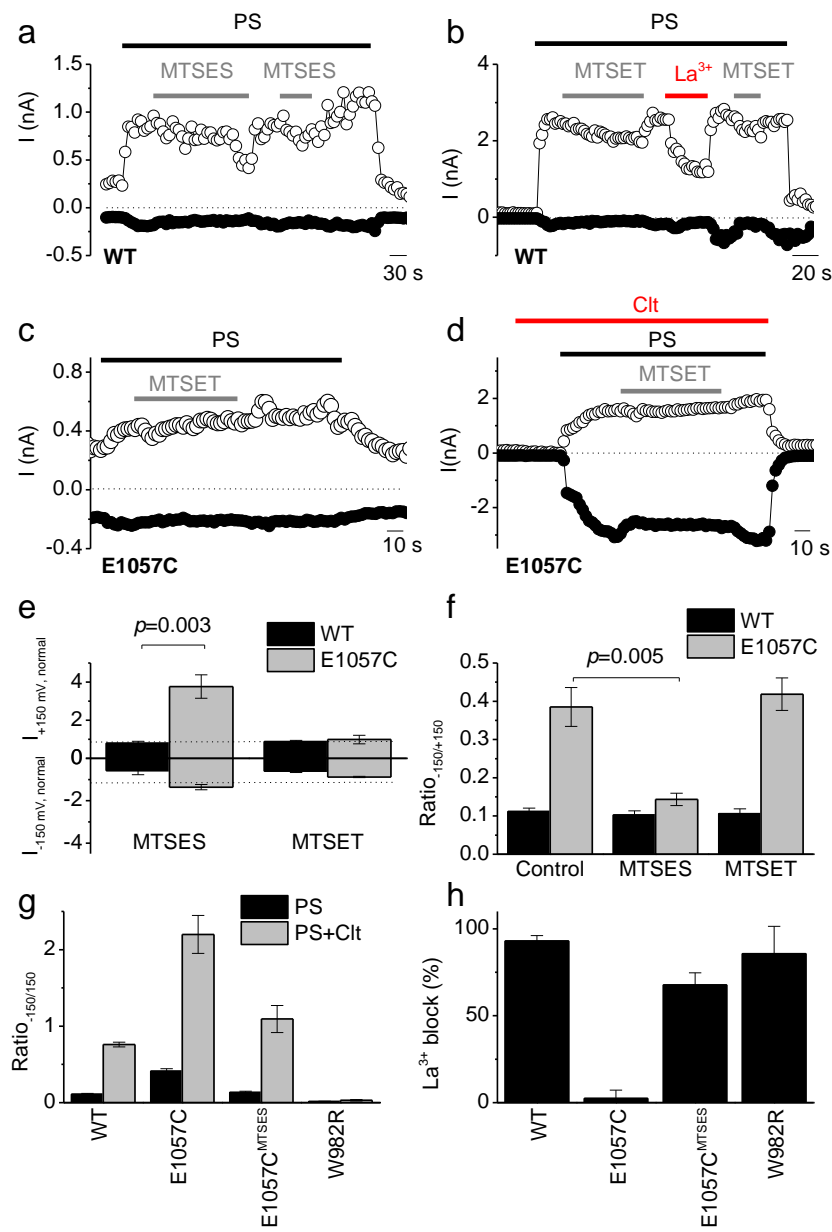

Supplementary Figure 9 Further properties of the substituted cysteine accessibility method on TRPM3 and the E1057C mutant. (a) Time course of whole cell currents at $\pm 80 \mathrm{mV}$ illustrating the lack of irreversible effect of MTSES (1 mM) on TRPM3 after stimulation by PS $(40 \mu \mathrm{M})$. (b) Time course of whole cell currents at $\pm 80 \mathrm{mV}$ of WT TRPM3 after stimulation by PS. No effect of MTSET (1 mM) on TRPM3 was observed. (c) Time course of whole cell currents $\pm 80 \mathrm{mV}$ illustrating the lack of effect of MTSET (1 mM) on the E1057C mutant channel. (d) Time course of whole cell currents $\pm 80 \mathrm{mV}$ of HEK 293 cells transiently transfected by E1057C mutant, activated by Clt $(10 \mu \mathrm{M})+\mathrm{PS}(40 \mu \mathrm{M})$, illustrating no potentiating effect of MTSET $(1 \mathrm{mM})$. (e) Statistics of MTSES and MTSET effect on the inward $(-150 \mathrm{mV})$ and outward $(+150 \mathrm{mV})$ current of WT TRPM3 (black bars) and E1057C mutant (gray bars). Currents are normalized to the current amplitude before application of 
MTSES/MTSET. ( $p=0.003$ for $n=5$ different experiments, unpaired t-test). (f) Ratio of the inward $(-150 \mathrm{mV})$ and outward $(+150 \mathrm{mV})$ current in control condition, and after stimulation by MTSES and MTSET in stable expressing TRPM3 cells (black bars) and transiently transfected E1057C HEK cells (gray bars) $(p=0.005$ for $\mathrm{n}=5$ different experiments, paired $\mathrm{t}-$ test). (g) Ratio $-150 /+150$ for currents activated by PS and PS+Clt for WT (n=84) and mutant channels (n=6-13). E1057C ${ }^{\text {MTSES }}$ represents the E1057C mutant after treatment with MTSES. (h) Percentage block by $\mathrm{La}^{3+}(100 \mu \mathrm{M})$ of the outward current activated by PS in WT $(\mathrm{n}=36)$ and mutant channels $(n=6-13)$. 

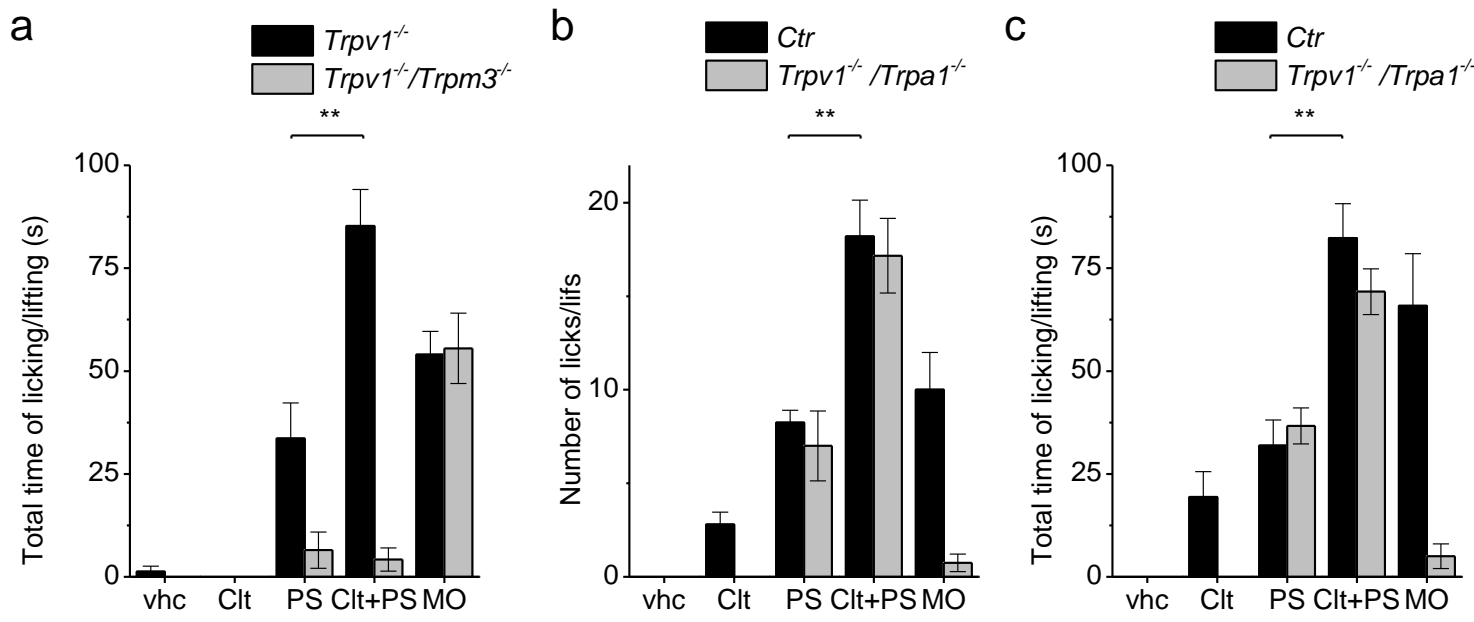

Supplementary Figure 10 Behavioral responses. (a) Total time spent on nocifensive behavioral following intraplantar injection of vehicle (Vhc), Clt, PS, PS+Clt, or mustard oil (MO) in $\operatorname{Tr} p v 1^{-/}$and $\operatorname{Tr} p v 1^{-/} / \operatorname{Tr} p m 3^{-/-}$mice (n=8 for each genotype; and **, $p=0.008$; paired $t$-test). (b) Number of behavioral responses (paw licks and lifts) and (c) total time spent on nocifensive behavioral following intraplantar injection of vehicle (Vhc), Clt, PS, PS+Clt, or mustard oil (MO) in wild-type and $\operatorname{Tr} p v 1^{-/} / \operatorname{Tr} p a^{-/-}$mice ( $\mathrm{n}=8$ for each genotype, $p=0.003$ in both cases, paired $t$-test). 

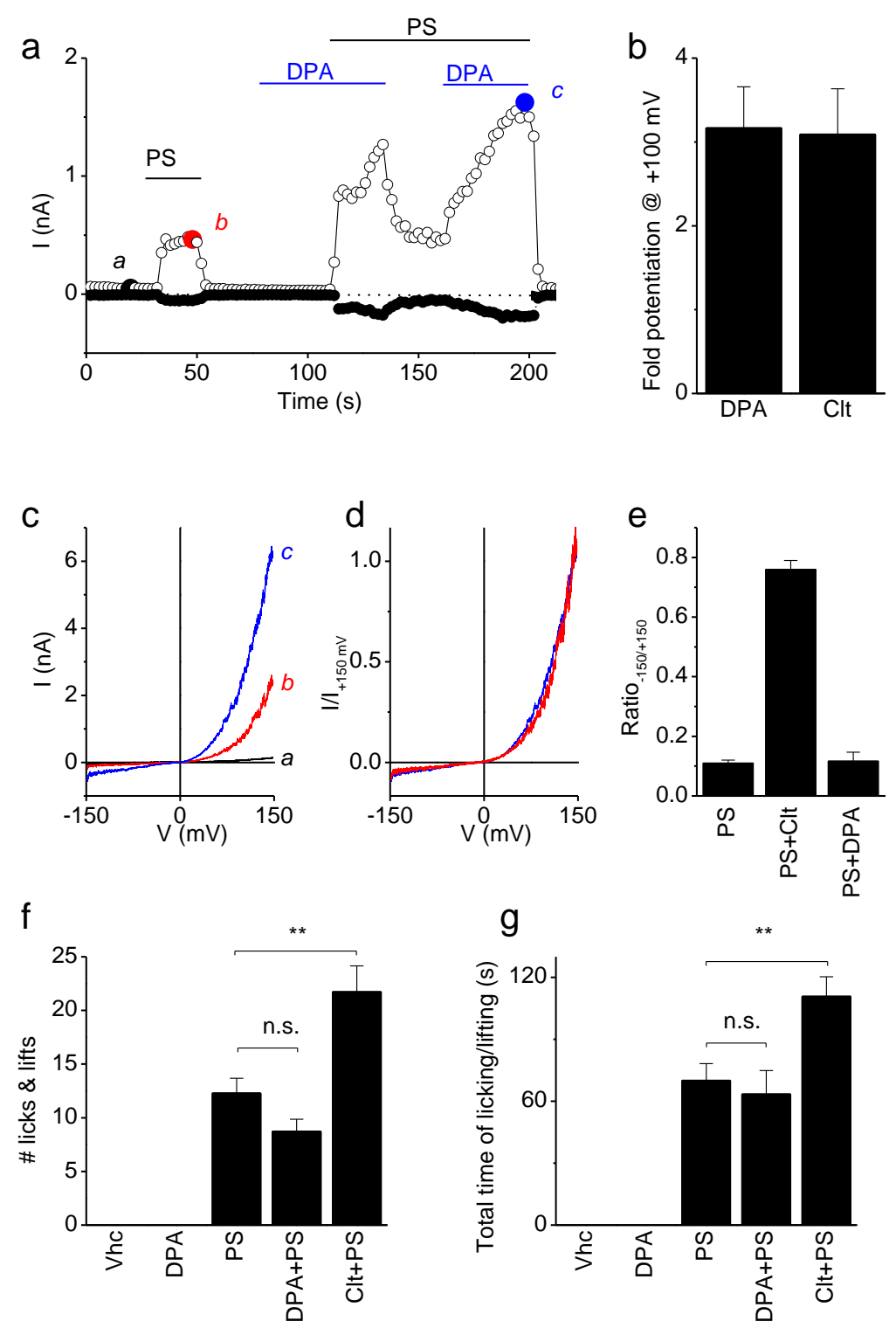

Supplementary Figure 11 Diphenylamine potentiates PS-activated TRPM3 currents. (a) Time course of whole-cell currents in TRPM3-expressing HEK293 cells at $\pm 80 \mathrm{mV}$ upon stimulation with PS $(40 \mu \mathrm{M})$ and diphenylamine (DPA, $10 \mu \mathrm{M})$. (b) Relative potentiation of the outward current at $+100 \mathrm{mV}$ upon stimulation with Clt $(10 \mu \mathrm{M} ; \mathrm{n}=48)$ and diphenylamine (DPA, $10 \mu \mathrm{M}, \mathrm{n}=7$ ). (c) I-V relations obtained at time points indicated in a. (d) Normalized I$\mathrm{V}$ relations showing the lack of effect of DPA on current rectification. (e) Values for ratio. 150/+150 for currents activated by PS in the absence or presence of Clt or DPA. (f) Number of 
behavioral responses (paw licks and lifts), and (g) duration of nociceptive behavior following intraplantar injection of vehicle (Vhc), diphenylamine (DPA), PS, PS+DPA, PS+Clt (n=7; **, $p=0.001$ in both tests; paired $t$-test). 

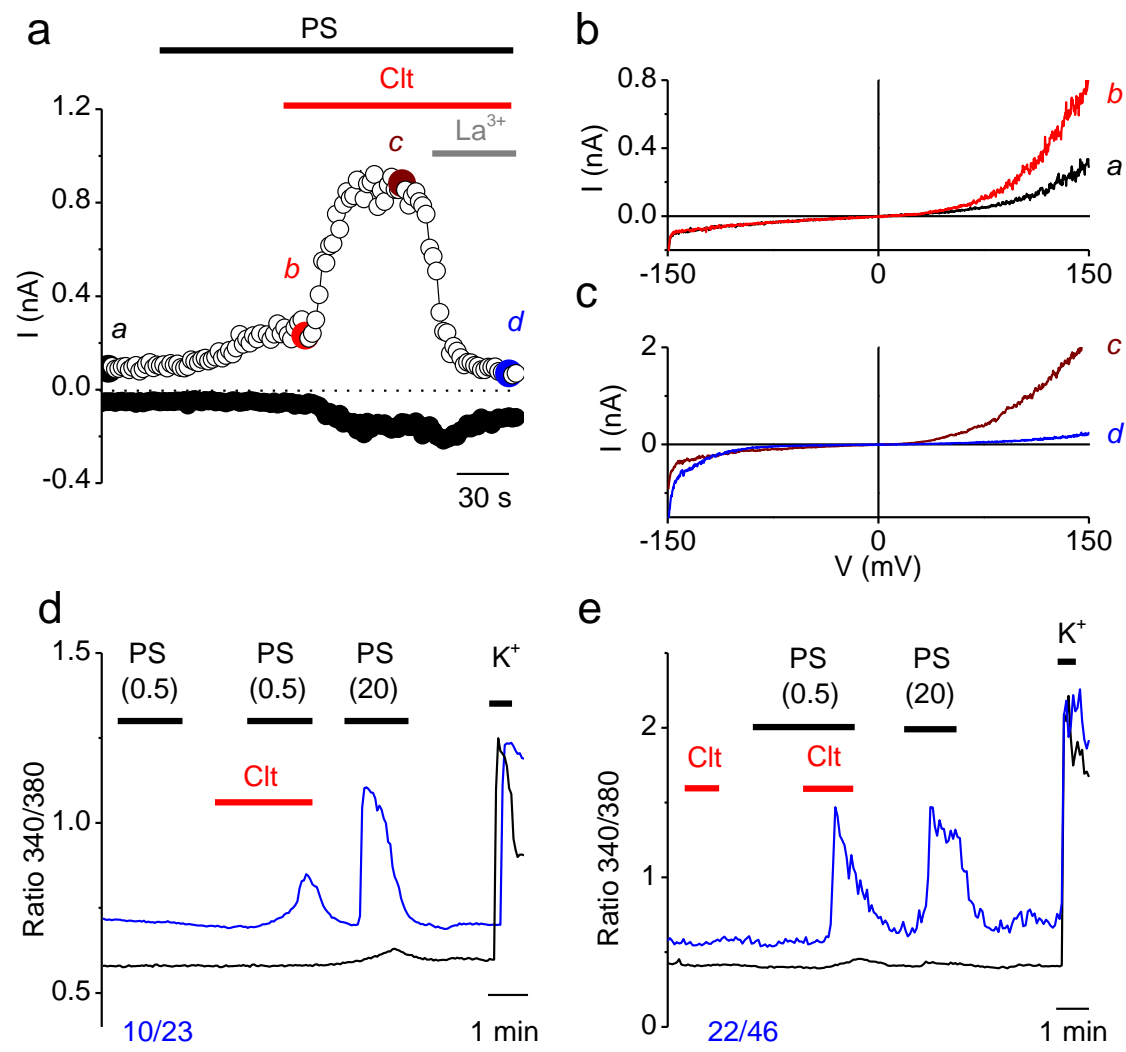

Supplementary Figure 12 Activation of the alternative pathway at $37^{\circ} \mathrm{C}$. (a) Time course of whole-cell TRPM3 currents at $\pm 80 \mathrm{mV}$ measured at $37{ }^{\circ} \mathrm{C}$, upon stimulation with PS (500 $\mathrm{nM})$ and Clt $(10 \mu \mathrm{M})$, and block by $\mathrm{La}^{3+}(10 \mu \mathrm{M})$. Representative example of 5 similar experiments. (b,c) I-V relations obtained at time points indicated in a. (d,e) Representative changes in intracellular $\mathrm{Ca}^{2+}$ concentration in DRG neurons derived from TRPV1 ${ }^{-/}$mice in response to PS $(500 \mathrm{nM}$ and $20 \mu \mathrm{M})$, Clt $(10 \mu \mathrm{M})$ and high $\mathrm{K}^{+}(50 \mathrm{mM})$ applied at $37{ }^{\circ} \mathrm{C}$. The numbers (blue) indicate the fraction of tested neurons that exhibited the specific response profile, namely a response to PS $(20 \mu \mathrm{M})$ and to the combination of Clt and PS (500 nM), but not to PS (500 nM) or Clt alone. 

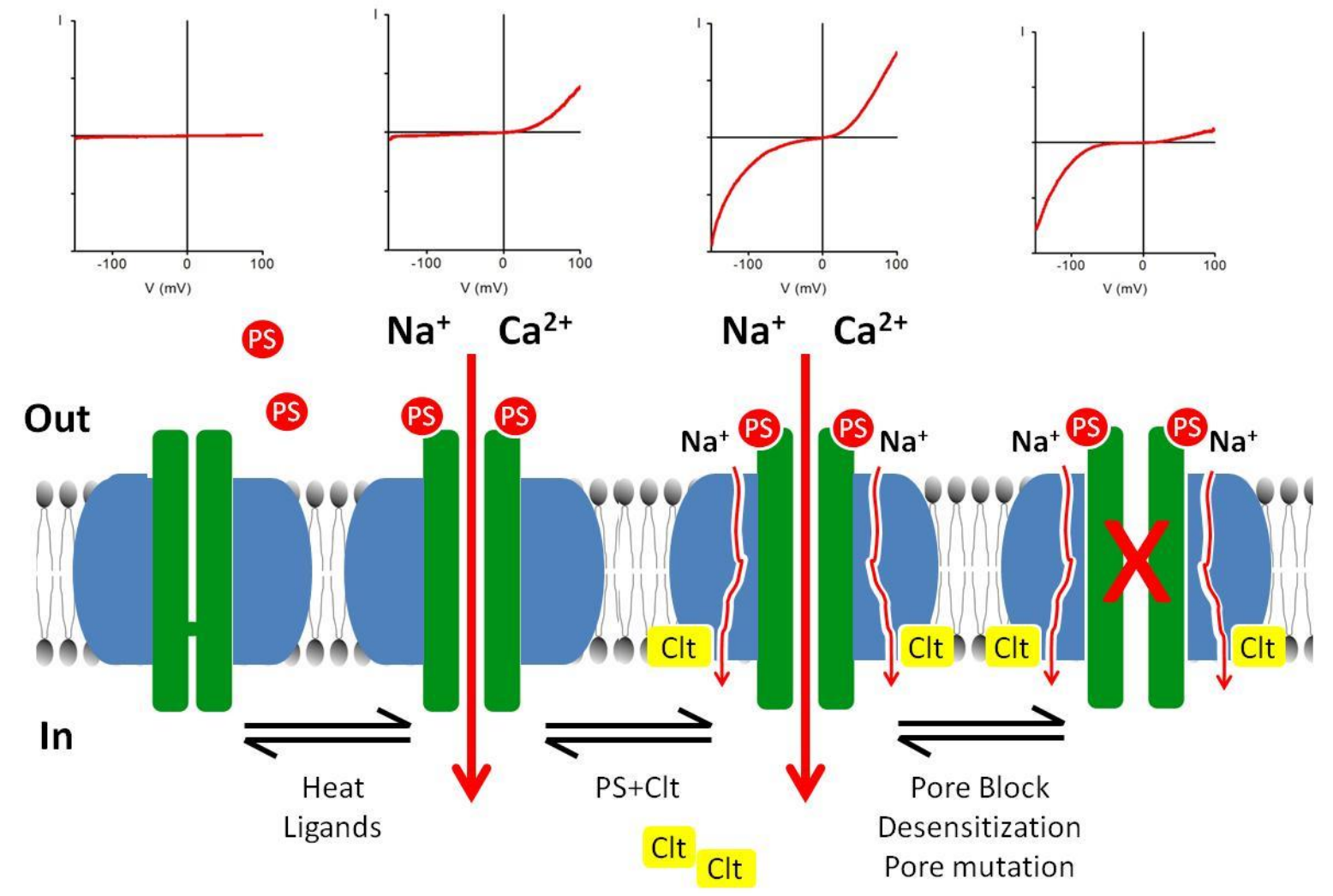

Supplementary Figure 13 Activation of an alternative cation permeation pathway in TRPM3. Cartoon illustrating the proposed mechanisms underlying activation of an alternative cation permeation pathway in TRPM3, distinct from the central pore. 